\title{
Computation of Forced Premixed Flames Dynamics
}

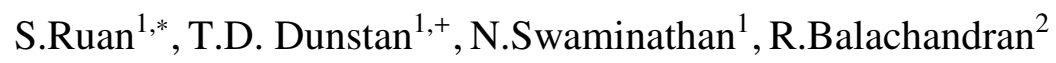 \\ 1 Engineering Department, Cambridge University, Trumpington Street, Cambridge \\ CB2 1PZ, UK. \\ ${ }^{2}$ Department of Mechanical Engineering, University College London, London WC1E \\ 7JE, UK.
}

*Corresponding author: S. Ruan. E-mail: sr468@cam.ac.uk

${ }^{+}$CurrentAddress:Met Office, FitzRoy Road, Exeter, Devon EX1 3PB, UK

Submitted for publication in Combustion Science and Technology

(First submitted July 27, 2015; Revised October 30 2015; Accepted 31 March 2016. ) 


\section{Abstract}

Bluff body stabilised turbulent premixed flames subject to inlet velocity oscillation over a wide range of forcing frequency and amplitude are simulated using a flameletbased combustion model. Two sets of detailed chemical kinetic schemes are used to model combustion chemistry. It is observed that the computed dynamics of forced flames agree reasonably well with experimental measurements. The flame elongation and shortening at a frequency of $40 \mathrm{~Hz}$ and strong flame-vortex interaction at a higher frequency of $160 \mathrm{~Hz}$ are captured well in the computations. The global flame describing function extracted from the computational results shows a linear response at $40 \mathrm{~Hz}$ and a nonlinear behaviour at $160 \mathrm{~Hz}$ as observed in the experiments. The nonlinear response is due to vortex roll-up and its subsequent shedding. The quantitative agreement of the computed flame describing function (FDF) with experimental measurement is uniformly good over a wide range of forcing frequency and amplitude. Some influence of chemical kinetics on the FDFs is observed, which mainly stems from the difference in laminar burning velocity and spatial heat release rate distribution.

Keywords: Turbulent premixed flame, Detailed chemistry effect, Flame Describing Function (FDF), combustion instability 


\section{Introduction}

Combustion instability has been the subject of many studies (McManus et al., 1993, Raun et al., 1993; Candel, 2002, Lieuwen, 2003) as it occurs in many practical devices such as rocket engines (Crocco, 1965), gas turbines (Lieuwen and Yang, 2005), ramjets (Roux et al., 2010) and afterburners (Bloxsidge et al., 1988). This is a process in which the fluctuations in pressure or velocity and heat release rate mutually influence one another leading to growth of these fluctuations. These growths can lead to self-sustained noisy combustion or damage the combustion system if the magnitude of this pressure fluctuation is exponentially large. Passive or active control, including closed and open-loop strategy, is commonly used to mitigate these issues in practical systems. Fundamental insights into the combustion instability are usually gained by studying the response of turbulent flame subject to a well characterised and controlled flow or pressure oscillations.

The combustion instability problem has been studied using theoretical (Lieuwen, 2003, Fleifil et al., 1996; Dowling, 1997, 1999; Schuller et al., 2003; Noiray et al., 2008), experimental (Ducruix et al., 2000; Lee and Santavicca, 2003; Balachandran et al., 2005) and numerical (Huang et al., 2003; Martin et al., 2006; Armitage et al., 2006; Han and Morgans, 2015) methods. One of the popular approach to investigate this problem in a complex practical system is the low-order modelling approach (Dowling and Stow, 2003; You et al., 2005), which considers the combustion system as a network of simple elements representing each part of the system. One crucial input for this kind of analysis is the "flame model" or "Flame describing Function" (FDF). . This function describes the flame (heat release rate) response to a velocity oscillation at a 
given frequency, $f$, and forcing amplitude, $A$, and it is defined as

$$
H(A, f)=\frac{Q_{f}^{\prime}}{u_{0, f}^{\prime}} .
$$

The normalised fluctuation of heat release rate in the combustion zone is defined as $Q^{\prime}=(Q-\bar{Q}) / \bar{Q}$, where $Q$ is the heat release rate at time $t$ and $\bar{Q}$ is the time averaged value. The symbol $u_{0}^{\prime}$ denote the normalised fluctuation of velocity at the inlet to the combustor. The subscript $f$ implies that these quantities are Fourier transformed and narrow-band filtered around the frequency $f$.

The FDF can be deduced through analytical studies (Lieuwen, 2003; Schuller et al., 2003; Noiray et al., 2008), experimental measurements (Ducruix et al., 2000; Paschereit et al., 2002; Lee and Santavicca, 2003; Balachandran et al., 2005) and numerical simulations (Lieuwen, 2003; Lieuwen and Yang, 2005; Huang and Yang, 2009; Lieuwen, 2012). Progress has been made including modelling forced flame dynamics (Krediet et al., 2012, Han and Morgans, 2015), combining Helmholtz solver with CFD to predict self-excited oscillations (Martin et al., 2006; Selle et al., 2006), stability analysis (Palies et al., 2011a; Silva et al., 2013), modelling of swirling flame transfer functions using analytical method (Palies et al., 2011b) and determination of FDFs for systems with multiple flames (Noiray et al., 2008; Boudy et al., 2011; Worth and Dawson, 2013). A comprehensive review of these studies is beyond the scope of this paper and readers are referred to recent reviews and the reference therein (Huang and Yang, 2009; Lieuwen, 2012). For computation of FDF using CFD, both Large Eddy Simulation (LES) (Menon and Jou, 1991; Möller et al., 1996; Angelberger et al., 2000; Huang et al., 2003; Martin et al., 2006; Selle et al., 2006; Staffelbach et al., 2009; Roux et al., 2010; Wolf et al., 2012; Krediet et al., 2012; Han and Morgans, 2015) and unsteady Reynolds Averaged Navier Stokes (URANS) (Smith and Leonard, 1997; 
Brewster et al., 1999; Steele et al., 2000; Brookes et al., 2001; Zhu et al., 2001, 2005; Armitage et al., 2006; Yao et al., 2012; Lee and Cant, 2013) paradigms have been used to study combustion instability in turbulent premixed flames. LES is especially suitable for calculating self-excited oscillation as its onset involves a complex interaction between heat release rate and the flow field. Nevertheless, URANS approach is still widely used in industry to help development of "silent" combustors for gas turbine application and this is mainly because of a huge difference in the computational costs for URANS and LES. It is likely that LES may be adopted for industry calculation as this cost difference may decrease with time. Nevertheless, URANS would be adequate to study the flame dynamics in response to a well characterised and deterministic velocity forcing as the flame reaches a periodic or cyclical state.

Although many previous numerical studies (Martin et al., 2006; Armitage et al., 2006; Han and Morgans, 2015) used simple one-step chemistry, the role of chemical kinetic modelling on the combustion instability characteristics has been recognised through a ramjet combustor LES (Roux et al., 2010) using one-step reactions with different kinetic parameters. Difference in the spatial distribution of heat release rate resulting from the chemical kinetics led to different combustion instability characteristics. Thus, this study aims to examine the role of multi-step chemical kinetics on the response of acoustically forced flames and their FDFs.

The first objective of this study is to assess the capability of a flamelet-based premixed combustion model using URANS methodology to capture the response of acoustically forced flame over a wide range of frequency and amplitude of the forcing. This assessment is conducted by comparing the computed flame response to those measured in Balachandran et al. (2005). The second objective is to study the role of chemical 
kinetic scheme on this flame response by using two detailed chemical kinetics mechanisms.

This paper is organised as follows. The modelling approach including combustion submodelling is described in section 2. The experimental test cases are described in section 3 and their numerical setup is discussed in section 4 . The results are presented in section 5 and conclusions are summarised in the final section.

\section{Modelling Methodology}

The averaged conservation equations for mass, momentum and energy (Swaminathan and Bray, 2011; Poinsot and Veynante, 2001) are solved. The Reynolds Stress model (RSM) (Launder, 1989) with a quadratic pressure-strain model (Speziale et al., 1991) is used for turbulence modelling. The standard forms of these transport equations can be found in the references cited above. Turbulent premixed combustion is modelled using a flamelet-presumed PDF (probability density function) approach as detailed next.

The transport equations for the Favre-averaged progress variable, $\widetilde{c}$, and its variance, $\widetilde{c^{\prime \prime 2}}$, are solved along with other governing equations noted above. The progress variable can be defined using temperature or sensible enthalpy or appropriate species mass fraction and a specific choice depends on the problem. The definition used for this study is given later in section 4 .

The transport equation for $\widetilde{c}$ is written as

$$
\frac{\partial \bar{\rho} \widetilde{c}}{\partial t}+\frac{\partial \bar{\rho} \widetilde{u_{k}} \tilde{c}}{\partial x_{k}}=\frac{\partial}{\partial x_{k}}\left(\overline{\rho D \frac{\partial c}{\partial x_{k}}}-\overline{\rho u_{k}^{\prime \prime} c^{\prime \prime}}\right)+\overline{\dot{\omega}}_{c}
$$


and for $\widetilde{c^{\prime \prime 2}}$ it is given by (Swaminathan and Bray, 2011; Poinsot and Veynante, 2001)

$$
\frac{\partial \bar{\rho} \widetilde{c^{\prime \prime 2}}}{\partial t}+\frac{\partial \bar{\rho} \widetilde{u_{k}} \widetilde{c^{\prime \prime 2}}}{\partial x_{k}}=\frac{\partial}{\partial x_{k}}\left(\overline{\rho D \frac{\partial c^{\prime \prime 2}}{\partial x_{k}}}-\overline{\rho u_{k}^{\prime \prime} c^{\prime \prime 2}}\right)-2 \overline{\rho u_{k}^{\prime \prime} c^{\prime \prime}} \frac{\partial \widetilde{c}}{\partial x_{k}}-2 \bar{\rho} \widetilde{\epsilon_{c}}+2 \overline{c^{\prime \prime} \dot{\omega}_{c}^{\prime \prime}}
$$

The symbol $D$ denotes the molecular mass diffusivity for $c$. The turbulent scalar fluxes in the above transport equations are modelled using gradient flux hypothesis following many earlier studies. Although the use of this hypothesis for turbulent premixed flames may be questionable, it generally holds for large turbulence level, typically $u_{\mathrm{rms}} / S_{L}^{0} \geq$ 4 , where $u_{\mathrm{rms}}$ is the root-mean-square value of turbulent fluctuation in unburnt mixture and $S_{L}^{0}$ is the burning velocity of unstrained planar laminar premixed flame. For this study, this approximation is seen as a first step for the current modelling framework and counter-gradient scalar flux can be included through appropriate transport equation. The validity of the gradient flux approximation used for flame conditions investigated here can be adjudged using results presented later in this paper.

The last two terms of Eq. (3) require modelling. The scalar dissipation rate defined as $\widetilde{\epsilon}_{c}=\overline{\rho D\left(\nabla c^{\prime \prime} \cdot \nabla c^{\prime \prime}\right)} / \bar{\rho}$, where $c^{\prime \prime}$ is the Favre fluctuation of $c$, is modelled here as Kolla et al. 2009)

$$
\widetilde{\epsilon}_{c} \simeq\left(\left[2 K_{c}^{*}-\tau C_{4}\right] \frac{S_{L}^{0}}{\delta_{L}^{0}}+C_{3} \frac{\widetilde{\varepsilon}}{\tilde{k}}\right) \frac{\widetilde{c^{\prime \prime 2}}}{\beta^{\prime}},
$$

where $\beta^{\prime}=6.7, C_{3}=1.5 \sqrt{\mathrm{Ka}} /(1+\sqrt{\mathrm{Ka}})$ and $C_{4}=1.1 /(1+\mathrm{Ka})^{0.4}$ are model parameters. The Karlovitz number is defined as $\mathrm{Ka}=t_{c} / t_{k}$, where the chemical time scale is defined as $t_{c}=\delta / S_{L}^{0}$ using the Zeldovich thickness $\delta$ and the Kolmogorov time scale is given by $t_{k}=\sqrt{v / \epsilon}$. The local kinematic viscosity of the reactant mixture is $v$ and $\widetilde{\epsilon}$ is the Favre-averaged dissipation rate of turbulent kinetic energy, $\widetilde{k}$. The heat release parameter is $\tau=\left(T_{b}-T_{u}\right) / T_{u}$, where $T$ is the absolute temperature and the subscripts $b$ and $u$ respectively denote the burnt and unburnt mixtures. The symbol $\delta_{L}^{0}$ is the thermal 
thickness of the unstrained planar laminar flame. The model parameter $K_{c}^{*}$ is taken to be $K_{c}^{*}=0.7 \tau$ for the hydrocarbon combustion considered in this study.

The unstrained premixed flamelet with a presumed shape is used to close the mean reaction rate and this model is written as

$$
\overline{\dot{\omega}}_{c}=\bar{\rho} \int_{0}^{1} \frac{\dot{\omega}_{c}(\zeta)}{\rho(\zeta)} \tilde{P}(\zeta) d \zeta
$$

where $\zeta$ is the sample space variable for $c, \dot{\omega}_{c} / \rho$ is from unstrained planar laminar flame calculation and $\tilde{P}(\zeta)$ is the marginal Favre PDF obtained using the Beta function for given values of $\widetilde{c}$ and $\widetilde{c^{\prime \prime 2}}$, which are computed using their respective transport equations. The contribution of chemical reaction related term, $\overline{c^{\prime \prime} \dot{\omega}_{c}^{\prime \prime}}$, in Eq. (3) is modelled as

$$
\overline{c^{\prime \prime} \dot{\omega}_{c}^{\prime \prime}} \approx \overline{c^{\prime \prime} \dot{\omega}_{c}}=\bar{\rho} \int_{0}^{1}(\zeta-\widetilde{\zeta}) \frac{\dot{\omega}_{c}(\zeta)}{\rho(\zeta)} \widetilde{P}(\zeta) d \zeta .
$$

The density and species mass fraction are obtained from integral expressions similar to Eq. (5). This flamelet model is appropriate to investigate combustion instability with frequency $f^{*}<1 / t_{c}$ as this inequality ensures that the flamelet structure is undisturbed by acoustic disturbance and the flamelet has enough time to respond to the imposed flow/acoustic variation within the forcing period. The above inequality roughly translates to $f^{*} \approx 6.5 \mathrm{kHz}$ for stoichiometric methane flame and typical frequencies observed in combustion instability are well below this limit.

The flamelet structure is obtained using freely propagating laminar premixed flame and thus an arbitrarily complex chemistry can be used while computing the flamelet structure. This allows us to study the influence of chemical mechanism on the characteristics of combustion instability. The above modelling methodology is used to simulate turbulent premixed flames stabilised behind a bluff-body and these experimental 
flames are described briefly next.

\section{Experimental Test Case}

The nonlinear response of turbulent premixed flame stabilised behind a bluff-body and subject to velocity forcing at the inlet was investigated for a wide range of forcing frequency and amplitude in (Balachandran et al. 2005). A schematic of this burner setup is illustrated in Fig. 1 1 showing that the burner consists of a long tube and a conical bluff body, supported by a rod, having a blockage ratio of 0.5 . Gaseous ethylene was injected into an air stream far upstream and a perfectly premixed mixture having an equivalence ratio of 0.55 was achieved. This mixture entered the burner tube as shown in Fig. 1 and bulk velocity oscillation was introduced using the loud speakers shown in this figure. The bulk-mean velocity in the annular exit in the plane of the bluff body base was $9.9 \mathrm{~m} / \mathrm{s}$, the bluff body diameter was $25 \mathrm{~mm}$ and these values gave a Reynolds number of about 19,000 . The frequency $f$ and amplitude $A$ of the velocity forcing were changed independently over wide ranges. Measurements of $\mathrm{OH}^{*}$ and $\mathrm{CH}^{*}$ chemiluminescence were performed by capturing the emission from the entire flame. Simultaneous $\mathrm{CH}_{2} \mathrm{O}$ and $\mathrm{OH}$ planar laser-induced fluorescence (PLIF) imaging was also performed over a smaller region of size $\mathcal{S}^{*}$ marked in Fig. 2 b). The Flame Surface Density (FSD) was then estimated using these OH-PLIF images and the local heat release rate was estimated using $[\mathrm{OH}] \times\left[\mathrm{CH}_{2} \mathrm{O}\right]$ signal. In this experiment, a non-linear flame response was observed for large values of $f$ with sufficiently high amplitude and thus these forced premixed flames offer a good challenge for modelling as it involves a complex interaction between turbulent flow and flames dynamics. Modelling of these flames were attempted in the past using a modified Bray-Moss-Libby (BML) and FSD 
approach (Armitage et al., 2006; Han and Morgans, 2015). In this study, flamelet approach described in the previous section is employed and the CFD (computational fluid dynamics) setup for computations of these experimental flames are described next.

\section{CFD Setup}

The combustion model discussed earlier in section 2 was implemented in a commercial CFD package FLUENT as described in (Chen et al., 2015; Ruan et al., 2015). This CFD package solves the necessary transport equations using finite volume methodology along with SIMPLE algorithm for pressure-velocity coupling, including the density variations in space and time. User defined scalar (UDS) were used to include the transport equations in Eqs. (2) and (3) for $\widetilde{c}$ and $\widetilde{c^{\prime \prime 2}}$ respectively. The various sources and sinks were included using user-defined-functions (UDFs). The detail of these procedure can be found in (Ruan et al., 2014, Chen et al., 2015).

Because of axisymmetric nature of averaged flow and flame, and there was no azimuthal instability present in the experiments (Balachandran et al., 2005), a two dimensional axisymmetric unstructured grid with 33,000 cells as depicted in Fig. 2 was used for the simulations using unsteady RANS methodology. The perturbations introduced by upstream velocity forcing were taken to be axisymmetric in this numerical treatment. The computational domain, $\mathcal{S}$, extended axially from $10 \mathrm{~mm}$ upstream of the bluff body to $80 \mathrm{~mm}$ downstream of it, and radially to $37.5 \mathrm{~mm}$ including the walls as shown in Fig. $2 \mathrm{a}$. The mesh was refined dynamically in shear and near-wall regions where the gradients of velocity, temperature and progress variable were large. The same grid was used for the range of forcing frequency and amplitude investigated 
here. This grid gave good resolution for flame brush over a complete forcing cycle. The smallest numerical cell size was about $0.016 \mathrm{~mm}$. This grid was selected after a grid sensitivity study using a mesh with 140,000 cells, with smallest grid size of about $0.008 \mathrm{~mm}$, to ensure that the results presented here are not sensitive to the numerical grid.

The boundary conditions used for the simulations are also illustrated in Fig. 2 a. No slip and adiabatic condition are used for the wall. All the scalar are specified to have zero gradient on the wall. Non-equilibrium wall function (Kim and Choudhury, 1995) are used to include pressure gradient effects in the near wall region. The velocity at the inlet was specified as

$$
U(t)=\bar{U}[1+A \sin (2 \pi f t)]
$$

with $\bar{U}=9.9 \mathrm{~m} / \mathrm{s}$. The mean progress variable, $\widetilde{c}$, and its variance, $\widetilde{c^{\prime \prime 2}}$, are set to zero at the inlet and have zero gradient at the pressure outlet. The turbulent intensity at the inlet is kept to a constant value of $5 \%$ when the mean velocity is varied. The progress variable is defined using temperature as $c=\left(T-T_{u}\right) /\left(T_{b}-T_{u}\right)$ for this study.

The combustion chemistry is represented using two chemical mechanisms USC (Wang and Laskin, 1998) and UCSD (UCSD, 2011) listed in Table 1 to investigate the effects of chemical kinetics on the flame response and FTFs. The USC mechanism involves 75 species and 529 elementary reactions, and the UCSD mechanism has 244 reactions and 50 species. A freely propagating laminar premixed flame of ethylene-air mixture having an equivalence ratio of 0.55 are computed using these two mechanisms first. Then, these results are used to construct a two dimensional look-up table containing the unclosed terms as described in section 2 and the controlling parameters for this look-up table are $\widetilde{c}$ and $g_{c}=\widetilde{c^{\prime \prime 2}} /[\widetilde{c}(1-\widetilde{c})]$. These two parameters vary between 0 and 
1, and are discretised using non-evenly distributed 21 points for each parameter with more resolution close to $\widetilde{c}$ with large reaction rate. These two controlling parameter values at a given grid point in the unsteady RANS simulations are obtained from their respective transport equations and a bi-linear interpolation with errors less than $1 \%$ are used to obtain the various sources and sinks from the look-up table.

A steady simulation is performed first using a constant bulk velocity by setting $\widetilde{c}=1$ everywhere inside the computation domain. After a steady flame is established, the bulk velocity is varied according to Eq. (7). For each forcing case, 100 time steps are used to simulate one complete forcing cycle and 8 complete cycles are calculated starting from the steady flame solution. Results from the last cycle are used to calculate the Flame Describing Functions for comparison with the experimental measurements.

\section{Results and Discussion}

\subsection{Unforced Flame}

Figure 3 compares the averaged FSD, $\Sigma$, obtained from the experiments and the mean reaction rate computed using both chemical mechanisms for the unforced case through $\overline{\dot{\omega}}_{c} \sim \rho_{u} S_{L}^{0} \Sigma$. The averaged FSD images shown here, were obtained using 100 instantaneous PLIF images, have maximum FSD in the range of $14-20 \mathrm{~cm}^{-1}$. This gives mean reaction rates close to the computed values. This agreement is encouraging but one must be cautious because the FSD is estimated using the flame contour length using a 2D cut and from neither reaction rate nor full 3D flame surface. The correspondence between the measured and computed flame shape, location and relative sizes are impor- 
tant for the analysis of this study. From the experimental measurements, it is clear that the flames formed downstream of the bluff body are not of equal strength. Two flames anchored on the bluff body are formed - one along the inner shear layer and the other along the outer shear layer, with the inner flame relatively stronger than the outer one. The inner flame is supported by hot products inside the recirculation zone downstream of the bluff body. The relatively weaker flame in the outer shear layer is because of cooler recirculation zone there. The flames impinge on the wall where the flame brush is thickened and reaction is weakened. These are likely due to the heat loss along the wall. Two distinct flames along the inner and outer shear layers are observed for the computational results obtained using both mechanisms as shown in Fig. 3. (b) and both of these reaction zones have similar level of reaction rate. For the UCSD mechanism, it gives a shorter flame brush compared to the USC mechanism. This is likely due to the different laminar flame speed yielded by the two mechanisms as shown in Table 1. A higher laminar flame speed of $21 \mathrm{~cm} / \mathrm{s}$ and a thinner flame is obtained with the UCSD mechanism, compared to $16.2 \mathrm{~cm} / \mathrm{s}$ and a thicker flame for the USC mechanism. Despite this difference in $S_{L}^{0}$, the flame structure in terms of temperature and major species variations obtained using these two mechanisms are very similar and they are consistent with previous study (Delfau et al., 2007) comparing lean ethylene flame structure computed using these two mechanisms and experimental measurements. Also, a previous study (Xu and Konnov, 2012) showed that the laminar flame speeds produced by these two mechanisms were within the scatter of many past experimental measurements. Figure. 4 presents the upper and lower bounds of experimental measurements together with $S_{L}^{0}$ computed using the USC and UCSD mechanisms over a range of equivalence ratio. Both mechanisms yield reasonable flame speed values.

The influence of chemical mechanisms on the turbulent flow field are shown in Fig 5 
where radial variation of turbulent kinetic energy at $10 \mathrm{~mm}$ downstream of the bluff body exit is presented. This position is selected as the difference in turbulent quantities begin to appear. There is only a small difference in central region, which is negligible. It is important to establish this basis because the bluff body exit velocity is used later in the analysis of Flame Describing Function. Although turbulence fluctuation does not appear explicitly in the FTF, it can alter the turbulent mixing between cold reactants and hot product, and thus the heat release rate which drives the FTF. The comparison of FTFs obtained using different mechanisms is meaningful only if the turbulence in the forced incoming flows in both cases are not significantly different.

The chemical mechanisms are likely to affect the turbulent flame speed, the speed at which the leading edge of the flame brush advances into the unburnt mixture relative to the incoming flow velocity. This speed can be estimated using KPP theory (see (Kolla et al., 2010) for correct reference), which suggests it is proportional to the square root of the gradient of mean reaction rate in the progress variable space as $\tilde{c}$ approach zero,

$$
S_{T} \sim \lim _{\tilde{c} \rightarrow 0} \sqrt{\frac{\partial \overline{\dot{\omega}}}{\partial \tilde{c}}}
$$

Figure 6 shows scatter plot of the mean progress variable against the mean reaction rate for the two mechanisms within the flame brush. The scatter data are collected from the steady simulations along the flame brush. The conditional mean $<\overline{\dot{\omega}}_{c} \mid \tilde{c}>$ is also included in the figures. An estimate of square root of the gradient as $\tilde{c}$ approach zero in Eq. 8) can be obtained using the conditional mean $\left\langle\overline{\dot{\omega}}_{c}\right| \tilde{c}>$, giving 9.3 and 11.0 for the USC and UCSD mechanism respectively, indicating $S_{T}$ can differ by $15 \%$ as a result of the mechanisms. This compares to the difference of $22 \%$ on the laminar flame speed, suggests the influence of chemical kinetics on turbulent flame speed is reduced. 


\subsection{Forced Flame Dynamics}

A velocity at the exit of the bluff body is used as a reference to characterise the forced flame response. Figure 7 illustrates an example of the forcing velocity and the resulting heat release rate of the flame from the experiment and the present simulation for a forcing frequency of $160 \mathrm{~Hz}$ and an amplitude of 0.45 . In the figure, the $\mathrm{OH}^{*}$ chemilluminescence signal from the experiment (Balachandran et al., 2005) is presented, which is proportional to the heat release rate, $\overline{\dot{\omega}}_{f} \Delta \mathrm{H}$, where $\Delta \mathrm{H}$ is the heating value of the fuel and $\overline{\dot{\omega}}_{f}$ is the fuel consumption rate and is related to $\overline{\dot{\omega}}_{c}$. The reference velocity is normalised using the bulk mean velocity of $9.9 \mathrm{~m} / \mathrm{s}$ in the unforced case. The computed time series of heat release rate in the forced case is normalised using the unforced value. The temporal variation of $\overline{\dot{\omega}}_{c}$ from the simulation shown in Fig.7 closely follows the $\mathrm{OH}^{*}$ signal from the experiment, indicating the phase of the flame response have been well captured by the simulation.

In the experiments (Balachandran et al., 2005), detailed measurement of OH-PLIF, $\mathrm{CH}^{*}$ and $\mathrm{OH}^{*}$ are available for 40 and $160 \mathrm{~Hz}$ over a wide range of forcing amplitude. These two frequencies respectively correspond to Strouhal numbers of 0.1 and 0.4 using $\bar{U}$ and $D$. Here two cases, $f=40 \mathrm{~Hz}$ with $A=0.60$ and $f=160 \mathrm{~Hz}$ with $A=0.64$, are chosen for detailed analysis.

Figure 8 presents a comparison of mean reaction rate obtained from the simulations and phased averaged FSD from the experiments for the case with $f=40 \mathrm{~Hz}$ and $A=0.60$ over a complete forcing cycle. The results are shown only for phase angles where significant variation of the flame behaviour is observed and this is found to be from $120^{\circ}$ to $210^{\circ}$. Experimental FSD sequence shows that the two flames move 
towards the wall at $60^{\circ}$ and subsequently for $120^{\circ}$ to $180^{\circ}$ phase angles, the flame collapses along the wall making it shorter and weaker near the wall and outer shear layer regions. The flame brush starts to regain its initial length from $210^{\circ}$, although the upstream part of the outer flame is still very weak even at $240^{\circ}$ and the initial attributes of the flame brush is seen to be recovered at about $300^{\circ}$. Due to the limitation of window size, experimental observations of FSD behaviour downstream of $50 \mathrm{~mm}$ is not available. Simulation results offer more insight on the flame dynamics in the full domain. The flow bulk velocity variation modulates the flame surface area and large scale flow motion makes the flame shorter and then the flame is flapped toward the wall where it is deformed and forced to sweep along the wall. One difference is that the computed flame appears shorter than those measured in the cycle minima, as seen in Fig 8 at $180^{\circ}$. But in general, the flame dynamics throughout the cycle is captured qualitatively well in the simulation. The difference between experimental observation and computational results observed for unforced flame also appears in this forced flame, ie., the weaker flame in outer shear layer and along the wall observed in the experiments is not captured in the simulation since no heat loss to the wall is included in the CFD model.

Some differences arising from the chemical kinetics can also be observed. Firstly, for the USC mechanism, simulation shows that there is unburnt reactant exiting the outlet, notably in Fig. 8 at the beginning and end of the forcing cycle signified by the open tip. This is not observed when the UCSD mechanism is used. This is due to the lower laminar flame speed given by the USC mechanism noted earlier. Finally, the wall tends to have a bigger effect in deforming the flame shape during the cycle for longer flame produced by the USC mechanism as shown in Fig 8 for $210^{\circ}$ and $240^{\circ}$. This is not unexpected because the unforced flame produced by the USC mechanism is almost touching the wall as seen in Fig. 3 and for the forced case flame-wall interaction effect 
is more prominent than that obtained by the UCSD mechanism.

A similar comparison for $f=160 \mathrm{~Hz}$ and $A=0.64$ is shown in Fig. 9 for a phase angle interval of 40 degree through a complete forcing cycle. Double flames are observed along the inner and outer shear layers initially. At $40^{\circ}$, flame in the inner shear layer started to deform and subsequently rolls up at $80^{\circ}$. The outer flame also starts to deform and the two flames collapse in downstream region leading to flame annihilation event. The flames near the burner are then wrapped around by the vortices shed off by the burner leading to the "mushroom" feature of the two flames at $160^{\circ}$ as observed in the experiment. These structures grow in size and are convected downstream leading to their collapse on the walls at about $280^{\circ}$. The whole cycle would start again with a new vortex forming at the flame base. In general, the flame dynamics in various phase angles have been well captured in the simulations. Differences between simulation and experimental results for $160 \mathrm{~Hz}$ case are similar to those for $40 \mathrm{~Hz}$ case and are due to adiabatic wall conditions used in the simulation.

The influence of chemical kinetics for $160 \mathrm{~Hz}$ case is as follows. For both simulations, there are flame annihilations leading to flame pinch-off from the "mushroom" structure. These unburnt mixture pockets are convected downstream and are completely consumed within the computational domain when the UCSD mechanism is used. For the USC mechanism, these pockets seem to be convected out of the domain (see Fig at $120^{\circ}$ ). Although not directly relevant here, it is worth noting that these hot spots and their acceleration downstream can produce entropy wave influencing thermo-acoustic instability (Goh and Morgans, 2013). Also, the "mushroom” structure, say observed at $200^{\circ}$, tends to be smaller with less wrinkling for the UCSD mechanism compared to that observed using the USC mechanism. These differences arise due to stronger local 
reaction as a result of higher laminar flame speed leading to more robust flame brush for the UCSD mechanism.

\subsection{Flame Describing Function}

After qualitatively comparing computational results with experimental observations, the model performance is assessed further by comparing the measured and computed FTFs. In order to evaluate the FDF, the time averaged heat release rate and its fluctuations are required. The mean value $\bar{Q}$ can either be calculated from the entire computational volume $\mathcal{S}$ as illustrated in Fig. 2(a) or using the information from the observation window $\mathcal{S}^{*}$ corresponding to the PLIF imaging in the experiments as illustrated in Fig. 2 (b). The mean value observed using the smaller domain $\mathcal{S}^{*}$ is denoted as $\bar{Q}^{*}$ here. This yields two sets of fluctuating heat release rate.

Figure 10 presents the flame response amplitude $Q_{A}^{\prime}$, defined as half of the peak-totrough normalised heat release fluctuation within one forcing cycle, ie., $Q_{A}^{\prime}=0.5\left(Q_{\max }^{\prime}-\right.$ $Q_{\text {min }}^{\prime}$ ), together with the FDF magnitude, $|H|$, also known as the gain of the FDF, and the phase angle, $\angle(H)$, obtained from simulations using both chemical mechanisms for $f=40$ and $160 \mathrm{~Hz}$. The computed values are compared to the experimental results obtained using both $\mathrm{CH}^{*}$ and $\mathrm{OH}^{*}$ chemiluminescence. The simulation results obtained using the USC mechanism for the domain $\mathcal{S}^{*}$ corresponding to the PLIF window size used in the experiment are also included and marked as "USC small" in Fig. 10. Previous study using Large Eddy Simulation and Flame Surface Density modelling (Han and Morgans, 2015) are included wherever possible for comparison purpose.

The first column of Fig. 10 compares the measured and computed flame response am- 
plitudes $Q_{A}^{\prime}$ for two frequencies. An approximately linear response is observed in the experiments for $40 \mathrm{~Hz}$ over a range of forcing amplitude. Numerical simulations using both mechanisms give very similar results for $A<0.4$ and they compare well with measured values. For larger values of $A$, the $Q_{A}^{\prime}$ computed with the UCSD mechanism is about $10 \%$ higher than those obtained using the USC mechanism. This is because some unburnt mixture is exiting the computational domain when the USC mechanism is used as noted earlier suggesting that the heat release rate response is not capture completely. In general, the computational results obtained using both mechanisms are in very good agreement with experimental measurement.

For $160 \mathrm{~Hz}$ shown in Fig 10 , a nonlinear response is observed. Three distinct regimes of flame response can be observed. For low forcing amplitude of $A<0.2$, the flame response is linear. There is a plateau in the flame response for $0.2 \leq A \leq 0.4$ before the response rises again with increasing $A$ and saturating at about $A=0.55$. This is related to the flame dynamics shown in Fig. 9 involving mushroom like structures, as also noted in (Armitage et al., 2006). The flame flapping behaviour (not shown here) is observed for $A<0.2$ due to the large scale flow motion similar to the one observed in $40 \mathrm{~Hz}$ case in Fig. 8. For forcing amplitude of $A=0.20$, a weak flame wrinkling due to vortex roll-up and its subsequent convection downstream starts to occur in the experiment (Balachandran et al. 2005). This onset is observed to occur in the simulation with the USC mechanism for $A=0.15$ and the UCSD mechanism for $A=0.25$. As the forcing amplitude increases, the flame becomes more wrinkled as the vortex rolls up the flame leading to mushroom feature observed in Fig. 9. However, even at large forcing amplitude such as $A=0.65$, the URANS simulations are unable to completely capture the level of dramatic flame distortion observed in the experiments. For example in Fig. 9, the flame shape at $120^{\circ}$ shows multiple foldings in the inner 
shear layer downstream of the bluff body in the experiment while in the simulations with both chemical mechanisms, this multiple foldings are not well reproduced. This explains a gentler increase in the computed $Q_{A}^{\prime}$ and an earlier saturation compared to measurement. For example, both computed flames response amplitude start to saturate at forcing amplitude of $A \approx 0.4$ instead of $A \approx 0.6$ in the experiment. Simulations using the UCSD mechanism give about $20 \%$ higher values for the flame response compared to those obtained using the USC mechanism at large forcing amplitudes of $A \approx 0.64$, although $Q_{A}^{\prime}$ are still lower compared to experimental values.

The middle column of Fig. 10 compares the computed FDF magnitude with experimental values for $f=40$ (top row in the figure) and $160 \mathrm{~Hz}$. Reasonable agreements are observed for values computed using both chemical mechanisms and experimental results for both frequencies. There are some small under-estimates in the FDF magnitude for large forcing amplitudes as in Fig. 10 .

The FDF phase information is presented in the third column of Fig. 10 comparing measured and computed values. The experimental results show a very small phase variation for $40 \mathrm{~Hz}$ and a substantial variation for $160 \mathrm{~Hz}$. The computational results generally agree well for both frequencies as shown in the figure. While the UCSD mechanism seems to give better agreement for $40 \mathrm{~Hz}$, it is the opposite for $160 \mathrm{~Hz}$ case. It is worth noting that for both frequencies over the complete range of forcing amplitudes, the UCSD mechanism gives consistently smaller phase difference, i.e. closer to zero, than the USC mechansim. This is likely due to its higher flame speed and thus a shorter flame, so that the convective time for flow disturbance to reach the flame is shorter, i.e. the effect of flow variation on heat release rate is felt sooner. This is consistent with observation in the flame dynamics, for example, the smaller mushroom structure for 
the UCSD mechanism compared to the USC mechanism seen in Fig. 9 at $120^{\circ}$.

Another observation in Fig. 10 for the computed results is that the FDF obtained from the entire domain $\mathcal{S}$ consistently agree well with the experimental values compared to the results obtained from the truncated domain $\mathcal{S}^{*}$. For example, the truncated domain only reproduce the correct trend in $Q_{A}^{\prime}$ shown in the first column of Fig. 10, and gives large error for FDF magnitude and phase as seen in Fig.10. This illustrates the importance of including the complete flame information while obtaining the FDF for system level analysis and this may not be so for PLIF experiments due to limited size of the observation window for PLIF.

Comparing with previous results of LES using FSD combustion modelling approach (Han and Morgans, 2015), the current modelling approach in URANS gives reasonable result with reduced computational cost.

Figure 11 presents a comparison of computed and measured FDF magnitude for a specific forcing amplitude $A$ over a range of forcing frequency and Strouhal number. The experimental measurements show a peak FDF magnitude at about $150 \mathrm{~Hz}$ for all forcing amplitudes investigated. The computed results capture this peak frequency and the variation in FDF magnitude very well, but the magnitude is generally underestimated for lower frequencies. As discussed previously, this is due to some unburnt products that exit the computational domain at low frequency, causing a lower flame response amplitude $Q_{A}^{\prime}$. For higher frequency, the simulations tend to overestimate the magnitude for small forcing amplitudes, e.g. $A=0.15$, but the agreement is quite good for large forcing amplitude, e.g. $A=0.30$. This is consistent with the previous observation in Fig. 10 while examining the FDF for specific frequencies. Note that in Fig. 11, only results computed with the USC mechanism are shown. The influence of chemical ki- 
netics on the FDF comes through the $Q_{A}^{\prime}$ which is shown in the first column of Fig. 10 . The chemical kinetics effects mainly manifest through the difference in laminar flame speed yielding different flame length and spatial heat release rate distribution. These differences lead to different response to acoustic forcing.

A scatter plot of phase angle, in multiples of $\pi$, variation with forcing frequency and Strouhal number is shown in Fig. 12 for both experiments and results computed with USC mechanism. The experimental data shows a diagonal band of variation, the phase angle decreases from 0 to -1 as the frequency increase from $20 \mathrm{~Hz}$ to $120 \mathrm{~Hz}$. As $f$ increases further, the phase angle jumps to 1 and then decreases. This typical saw-tooth variation is well-captured in the simulations and the computational results typically falls within the experimental scatter for the phase angle for a specific frequency, which is due to different forcing amplitude. This shows that the global FDF phase angle variation with frequency is captured reasonably well in the simulations.

\section{Conclusion}

The response of turbulent premixed flames stabilised behind a bluff body and subjected to oscillations of bulk velocity is investigated in this study using unsteady RANS methodology over a wide range of forcing frequency and amplitude. An unstrained laminar flamelet model with presumed PDF shape is used to calculate the mean reaction rate. Combustion chemistry is modelled using two detailed chemical kinetic, UCSD and USC, mechanisms through tabulated chemistry approach to investigate the effects of chemical kinetic modelling on the dynamics of forced flames and their Flame Describing Function (FDF). 
It is observed that these simulations capture reasonably well the flame surface modulation by large scale flow oscillation at low frequency and the strong flame-vortex interaction at high frequency observed in experiments. Desipte this, the simulation does not include heat loss effect leading to some over prediction of heat release in the outer shear layer. Also, the flame-wall interaction needs further investigation to better rrepresent the flame deformation near the wall. The effect of chemical kinetics manifests through the difference in burning velocity yielding differences in flame length and spatial distribution of heat release rate. Also, the influence of chemical kinetics is observed to be small for the computed FDF. The computed FDF agrees reasonably well with the measured FDF over different frequencies and amplitude, despite the fact that FDF is under-predicted at high forcing amplitude. All of these will be addressed in a future study.

\section{Acknowledgment}

The support of Mitsubishi Heavy Industries, Takasago, Japan is acknowledged gratefully.

\section{References}

Angelberger, C., Egolfopoulos, F., and Veynante, D., 2000. Large eddy simulations of chemical and acoustic effects on combustion instabilities. Flow. Turbulence. Combust. 65, 205-220.

Armitage, C.A., Balachandran, R., Mastorakos, E., and Cant, R.S., 2006. Investigation 
of the nonlinear response of turbulent premixed flames to imposed inlet velocity oscillations. Combust. Flame 146, 419-436.

Balachandran, R., Ayoola, B.O., Kaminski, C.F., Dowling, A.P., and Mastorakos, E., 2005. Experimental investigation of the nonlinear response of turbulent premixed flames to imposed inlet velocityoscillations. Combust. Flame 143, 37-55.

Bloxsidge, G.J., Dowling, A.P., and Langhorne, P.J., 1988. Reheat buzz: an acoustically coupled combustion instability. part 2. theory. J. Fluid Mech. 193, 445-473.

Boudy, F., Durox, D., Schuller, T., and Candel, S., 2011. Nonlinear mode triggering in a multiple flame combustor. Proc. Combust. Inst. 33, 1121-1128.

Brewster, B.S., Cannon, S.M., Farmer, J.R., and Meng, F., 1999. Modeling of lean premixed combustion in stationary gas turbines. Prog. Engy. Combust. Sci 25, 353385.

Brookes, S., Cant, R., Dupere, I., and Dowling, A., 2001. Computational modeling of selfexcited combustion instabilities. J. Eng. Gas. Turbine. Power 123, 322-326.

Candel, S., 2002. Combustion dynamics and control: Progress and challenges. Proc. Combust. Inst. 29, 1-28.

Chen, Z., Ruan, S., and Swaminathan, N., 2015. Simulation of turbulent lifted methane jet flames: Effects of air-dilution and transient flame propagation. Combust. Flame $162,703-716$.

Crocco, L., 1965. Theoretical studies on liquid-propellant rocket instability. Proc. Combust. Inst. 10, 1101-1128.

Delfau, J.L., Biet, J., Idir, M., Pillier, L., and Vovelle, C., 2007. Experimental and numerical study of premixed lean ethylene flames. Proc. Combust. Inst. 31, 357365 .

Dowling, A.P., 1997. Nonlinear self-excited oscillations of a ducted flame. J. Fluid 
Mech. 346, 271-290.

Dowling, A.P., 1999. A kinematic model of a ducted flame. J. Fluid Mech. 394, 51-72.

Dowling, A.P., and Stow, S.R., 2003. Acoustic analysis of gas turbine combustors. $J$. Propul. Power 19, 751-764.

Ducruix, S., Durox, D., and Candel, S.., 2000. Theoretical and experimental determination of the transfer function of a laminar premixed flame. Proc. Combust. Inst. $28,765-773$.

Fleifil, M., Annaswamy, A., Ghoneim, Z., and Ghoniem, A., 1996. Response of a laminar premixed flame to flow oscillations: a kinematic model and thermoacoustic instability results. Combust. Flame 106, 487-510.

Goh, C.S., and Morgans, A.S., 2013. The influence of entropy waves on the thermoacoustic stability of a model combustor. Combust. Sci. Technol. 185, 249-268.

Han, X., and Morgans, A.S., 2015. Simulation of the flame describing function of a turbulent premixed flame using an open-source les solver. Combust. Flame 162, $1778-1792$.

Huang, Y., Sung, H.G., Hsieh, S.Y., and Yang, V., 2003. Large eddy simulation of combustion dynamics of lean-premixed swirl-stabilized combustor. J. Propuls. Power $19,782-794$.

Huang, Y., and Yang, V., 2009. Dynamics and stability of lean-premixed swirlstabilized combustion. Prog. Engy. Combust. Sci 35, 293-364.

Kim, S.E., and Choudhury, D., 1995. A near-wall treatment using wall functions sensitized to pressure gradient, in: ASME FED Vol. 217 Separated and Complex Flows. ASME.

Kolla, H., Rogerson, J.W., Chakraborty, N., and Swaminathan, N., 2009. Scalar dissipation rate modeling and its validation. Combust. Sci. Technol. 181, 518-535. 
Kolla, H., Rogerson, J.W., and Swaminathan, N., 2010. Validation of a turbulent flame speed model across combustion regimes. Combust. Sci. Technol. 182, 284-308.

Krediet, H.J., Beck, C.H., Krebs, W., Schimek, S., Paschereit, C.O., and Kok, J.B.W., 2012. Identification of the flame describing function of a premixed swirl flame from les. Combust. Sci. Technol. 184, 888-900.

Launder, B.E., 1989. Second-moment closure: Present... and future? Int. J. Heat Fluid Flow 10, 282-300.

Lee, C., and Cant, R., 2013. Cfd investigation of turbulent premixed flame response to transverse forcing, in: ASME Paper GT2013-94312.

Lee, J.G., and Santavicca, D.A., 2003. Experimental diagnostics for the study of combustion instabilities in lean premixed combustors. J. Propul. Power 19, 735-750.

Lieuwen, T., 2003. Modeling premixed combustion-acoustic wave interactions: A review. J. Propul. Power 19, 765-781.

Lieuwen, T., 2012. Unsteady Combustor Physics. Cambridge University Press, Cambridge, UK.

Lieuwen, T., and Yang, V. (Eds.), 2005. Combustion Instabilities in Gas Turbine Engines: Operational Experience, Fundamental Mechanisms, and Modeling. AIAA Inc.

Martin, C., Benoit, L., Nicoud, F., Poinsot, T., and Sommerer, Y., 2006. Large-eddy simulation and acoustic analysis of a swirled staged turbulent combustor. AIAA J. $44,741-750$.

McManus, K., Poinsot, T., and Candel, S., 1993. A review of active control of combustion instabilities. Prog. Engy. Combust. Sci 19, 1-29.

Menon, S., and Jou, W.H., 1991. Large-eddy simulation of combustion instability in and axisymmetric ramjet combustor. Combust. Sci. Technol. 75, 53-72. 
Möller, S., Lundgren, E., and Fureby, C., 1996. Large eddy simulation of unsteady combustion. Proc. Combust. Inst 26, 241-248.

Noiray, N., Durox, D., Schuller, T., and Candel, S., 2008. A unified framework for nonlinear combustion instability analysis based on the flame describing function. $J$. Fluid. Mech 615, 139-167.

Palies, P., Durox, D., Schuller, T., and Candel, S., 2011a. Nonlinear combustion instability analysis based on the flame describing function applied to turbulent premixed swirling flames. Combust. Flame 168, 1980-1991.

Palies, P., Schuller, T., Durox, D., and Candel, S., 2011b. Modeling of premixed swirling flames transfer functions. Proc. Combust. Inst. 33, 2967-2974.

Paschereit, C.O., Schuermans, B., Polifke, W., and Mattson, O., 2002. Measurement of transfer matrices and source terms of premixed flames. J. Eng. Gas Turbine. Power $124,239-247$.

Poinsot, T., and Veynante, D., 2001. Theoretical and Numerical Combustion. Edwards. Raun, R., Beckstead, M., Finlinson, J., and Brooks, K., 1993. A review of rijke tubes, rijke burners and related devices. Prog. Engy. Combust. Sci 19, 313-364.

Roux, A., Gicquel, L., Reichstadt, S., Bertier, N., Staffelbach, G., Vuillot, F., and Poinsot, T., 2010. Analysis of unsteady reacting flows and impact of chemistry description in large eddy simulations of side-dump ramjet combustors. Combust. Flame 157, 176-191.

Ruan, S., Swaminathan, N., and Darbyshire, O., 2014. Modelling of turbulent lifted jet flames using flamelets: a priori assessment and a posteriori validation. Combust. Theory. Modelling 18, 295-329.

Ruan, S., Swaminathan, N., Isono, M., Saitoh, T., , and Saitoh, K., 2015. Simulation of premixed combustion with varying equivalence ratio in gas turbine combustor. $J$. 
Propul. Power 31, 861-871.

Schuller, T., Durox, D., and Candel, S., 2003. A unified model for the prediction of laminar flame transfer functions : comparisons between conical and v-flame dynamics. Combust. Flame 134, 21-34.

Selle, L., Benoit, L., Poinsot, T., Nicoud, F., and Krebs, W., 2006. Joint use of compressible large-eddy simulation and helmholtz solvers for the analysis of rotating modes in an industrial swirled burner. Combust. Flame 145, 194-205.

Silva, C.F., Nicoud, F., Schuller, T., Durox, D., and Candel, S., 2013. Combining a helmholtz solver with the flame describing function to assess combustion instability in a premixed swirled combustor. Combust. Flame 160, 1743-1754.

Smith, C., and Leonard, A., 1997. Cfd modelling of combustion instability in premixed axisymmetric combustors, in: ASME Paper 97-GT-305.

Speziale, C.G., Sarkar, S., and Gatski, T.B., 1991. Modelling the pressurestrain correlation of turbulence: an invariant dynamical systems approach. J. Fluid. Mech 227, $245-272$.

Staffelbach, G., Gicquel, L., Boudier, G., and Poinsot, T., 2009. Large eddy simulation of self-excited azimuthal modes in annular combustors. Proc. Combust. Inst. 32, 29092916.

Steele, R., Cowell, L., Cannon, S., and Smith, C., 2000. Passive control of combustion instability in lean-premixed combustors. J. Eng. Gas Turbine. Power 122, 412-419.

Swaminathan, N., and Bray, K.N.C., 2011. Turbulent Premixed Combustion. Cambridge University Press, Cambridge, UK.

UCSD, 2011. Chemical-Kinetic Mechanisms for Combustion Applications. URL: http://web.eng.ucsd.edu/mae/groups/combustion/mechanism.html.

Wang, H., and Laskin, A., 1998. A Comprehensive Reaction Model of Ethylene and 
Acetylene Combustion. URL: http://ignis.usc.edu/Mechanisms/C2-C4/ c2.html.

Wolf, P., Balakrishnan, R., Staffelbach, G., Gicquel, L.Y.M., and Poinsot, T., 2012. Using les to study reacting flows and instabilities in annular combustion chambers. Flow. Turbulence. Combust. 88, 191-206.

Worth, N.A., and Dawson, J.R., 2013. Self-excited circumferential instabilities in a model annular gas turbine combustor: Global flame dynamics. Proc. Combust. Inst. 34, 3127-3134.

Xu, C., and Konnov, A.A., 2012. Validation and analysis of detailed kinetic models for ethylene combustion. Energy 43, 19-29.

Yao, Z., Gao, Y., Zhu, M., Dowling, A.P., and Bray, K.N.C., 2012. Combustion rumble prediction with integrated computational-fluid-dynamics/low-order-model methods. J. Propul. Power 28, 1015-1025.

You, D., Yang, V., and Sun, X., 2005. Three-dimensional linear stability analysis of gas turbine combustion dynamics, in: Lieuwen, T., and Yang, V. (Eds.), Combustion Instabilities in Gas Turbine Engines: Operational Experience, Fundamental Mechanisms, and Modeling. AIAA Inc.. chapter 14, pp. 415-444.

Zhu, M., Dowling, A.P., and Bray, K.N.C., 2001. Self-excited oscillations in combustors with spray atomizers. J. Eng. Gas Turbine. Power 3, 779-786.

Zhu, M., Dowling, A.P., and Bray, K.N.C., 2005. Transfer function calculations for aero-engine combustion oscillations. J. Eng. Gas Turbine. Power 127, 18-26. 


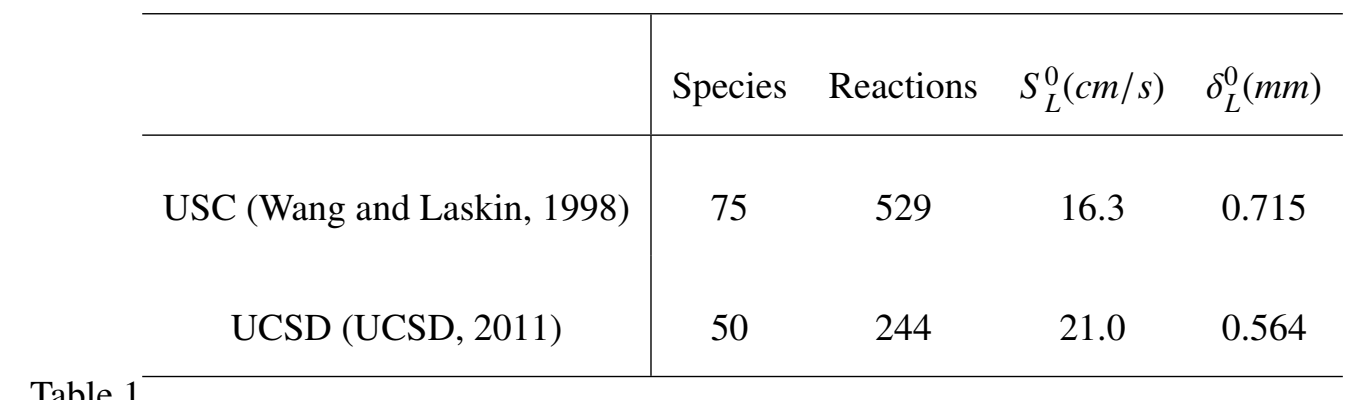

Table 1

Comparison of laminar flame quantities obtained using two detailed mechanisms for $\phi=0.55$ ethylene-air mixture.

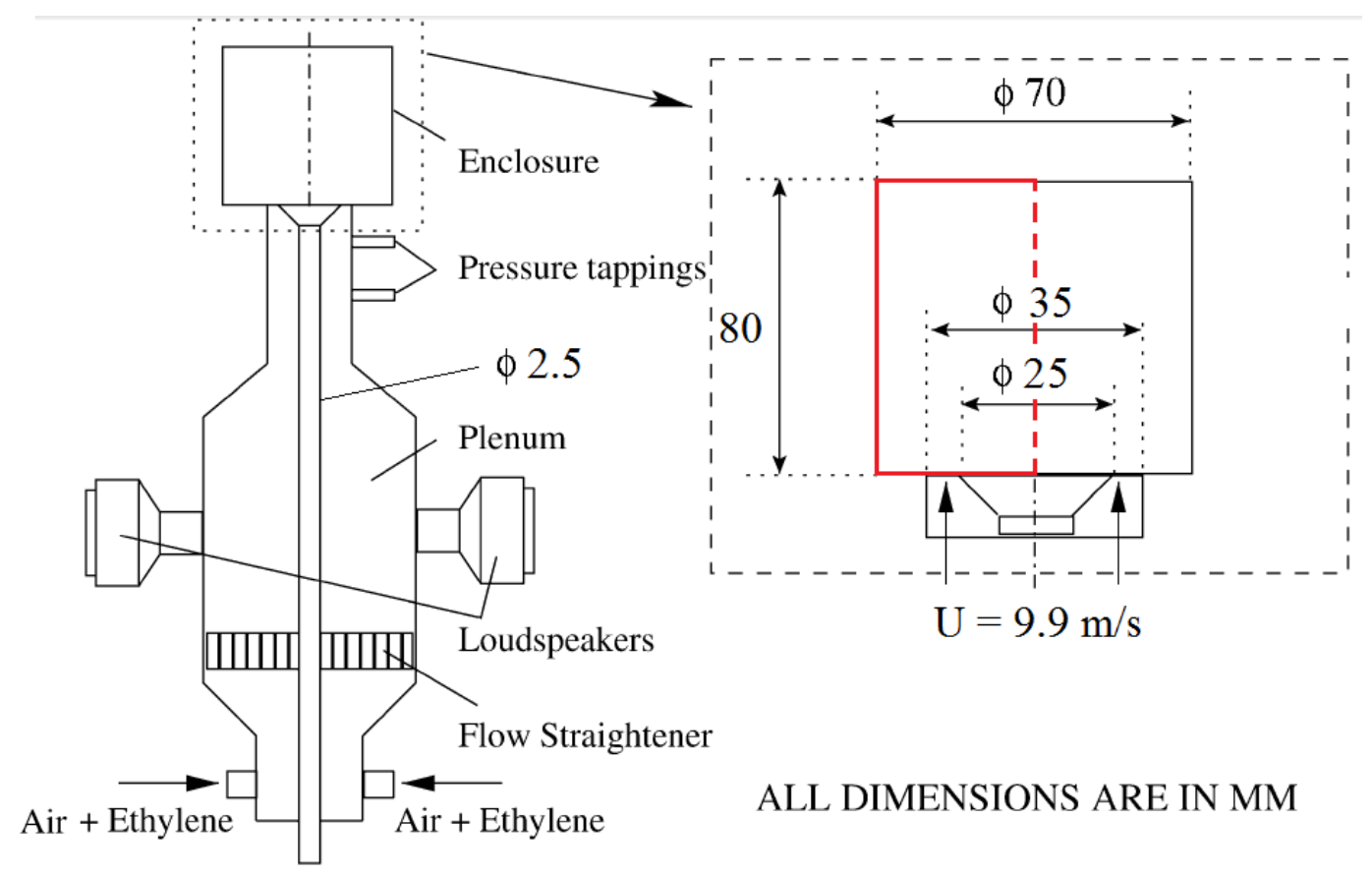

Fig. 1. Schematics of the experimental set up in (Balachandran et al., 2005) 


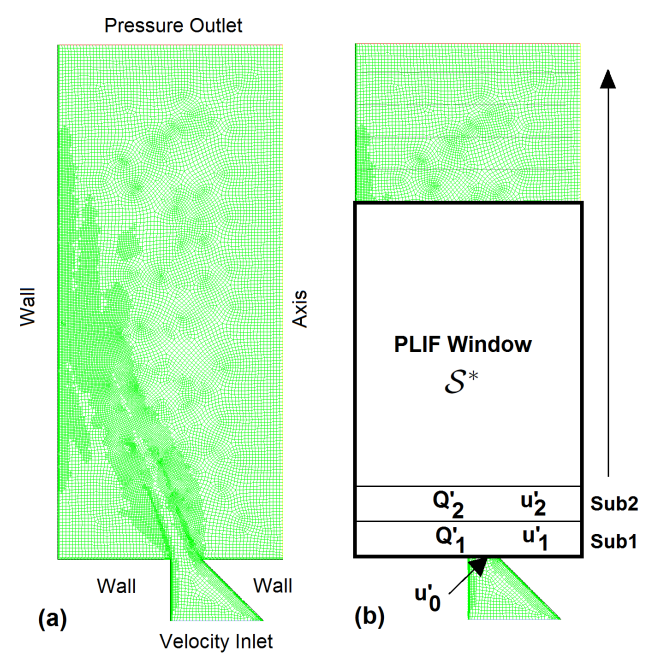

Fig. 2. (a) Computational Domain $\mathcal{S}$ with boundary condition. (b) The highlighted box corresponds to the PLIF measurement window $\mathcal{S}^{*}$ in the experiment (Balachandran et al., 2005). 


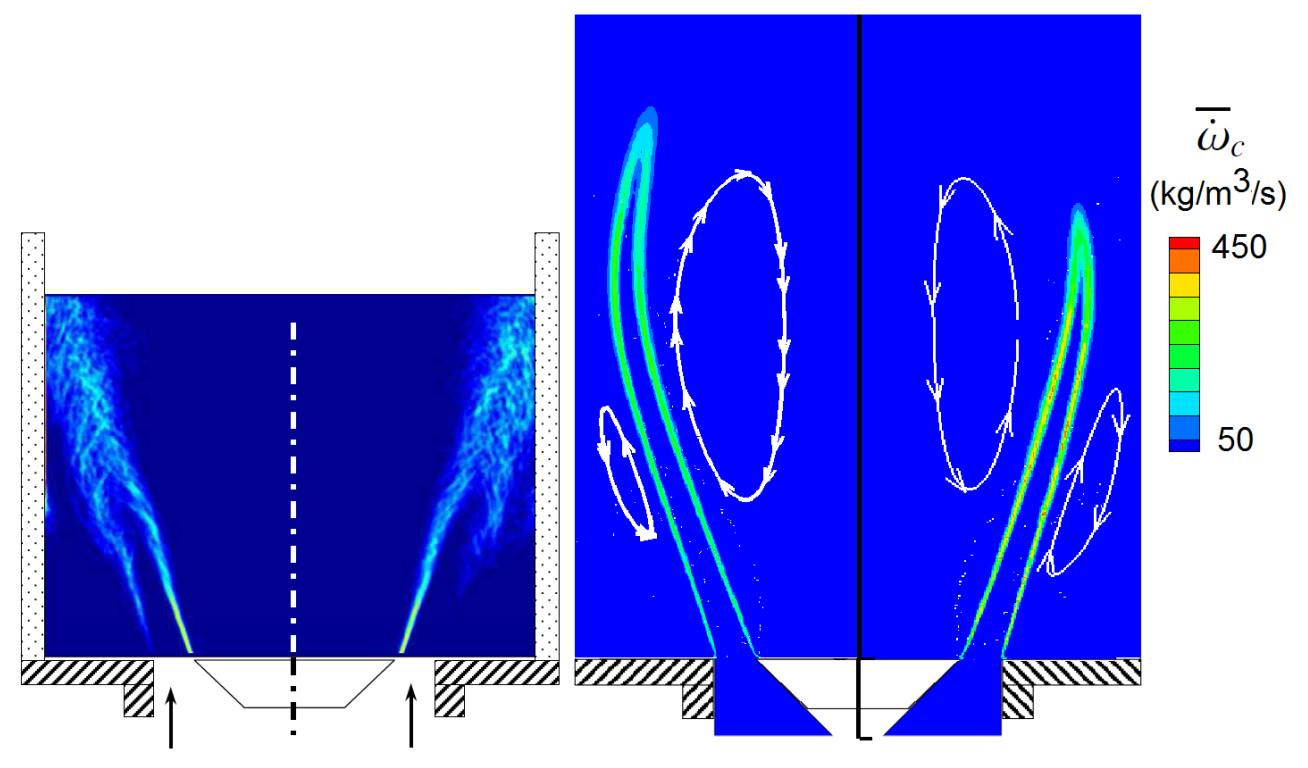

Fig. 3. Unforced flame results, comparison of FSD estimated using OH PLIF technique in a smaller domain $\mathcal{S}^{*}$ (left), and computed mean reaction rate $\overline{\dot{\omega}}_{c}$ with USC mechanism (middle) and UCSD mechanism (right) in full domain $\mathcal{S}$. 


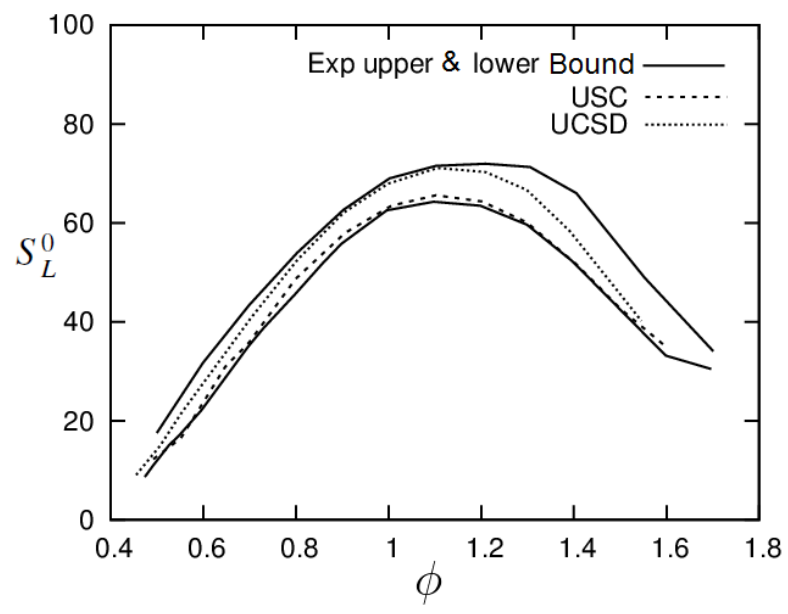

Fig. 4. Laminar flame speed $(\mathrm{cm} / \mathrm{s})$ against equivalence ratio for a collection of experimental measurements (Xu and Konnov, 2012), and calculations from USC and UCSD mechanisms. 


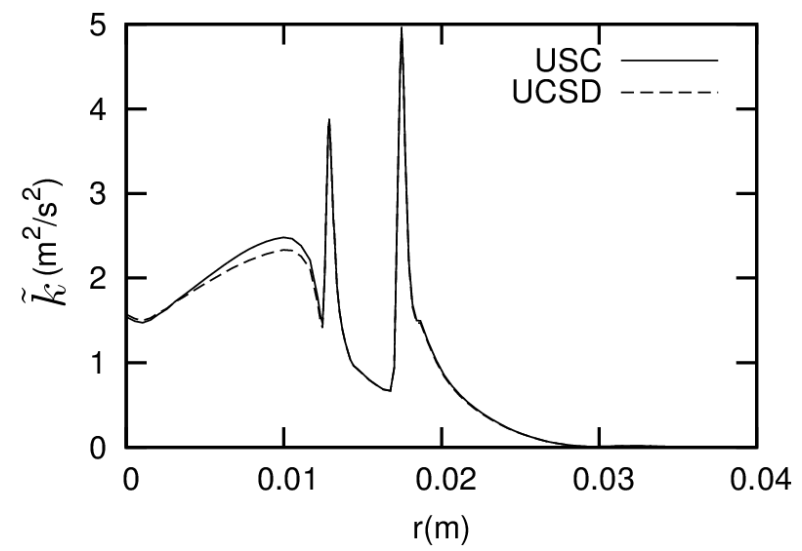

Fig. 5. Radial varaition of turbulence kinetic energy for two mechanisms at an axial position of $10 \mathrm{~mm}$
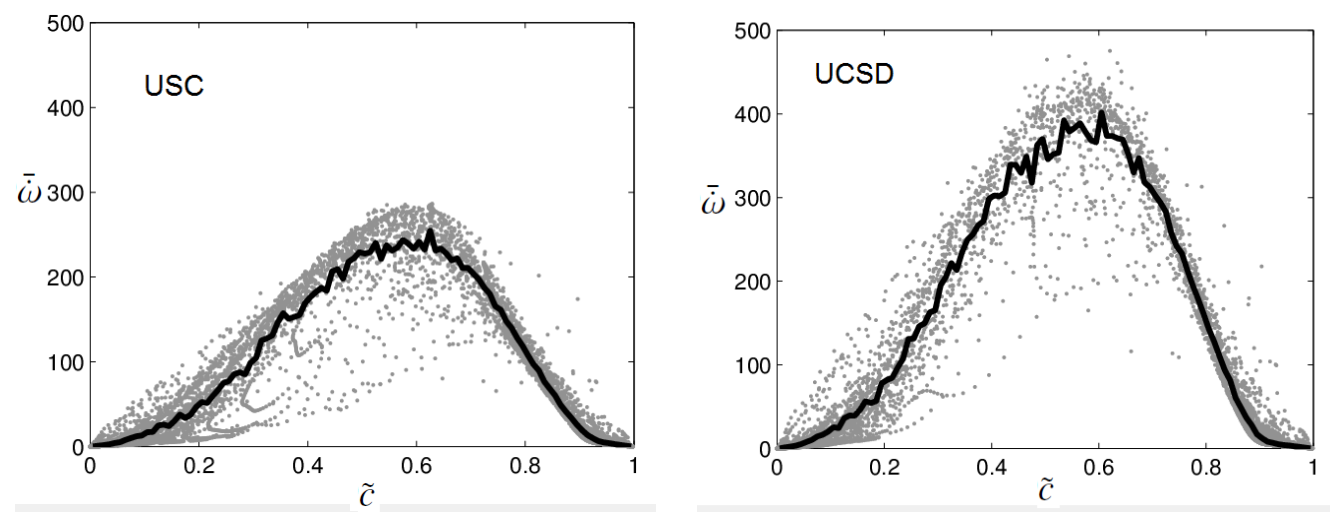

Fig. 6. Scatter plot of mean reaction rate $\left(\mathrm{kg} / \mathrm{m}^{3} / \mathrm{s}\right)$ against $\tilde{c}$ for different mechanism. 

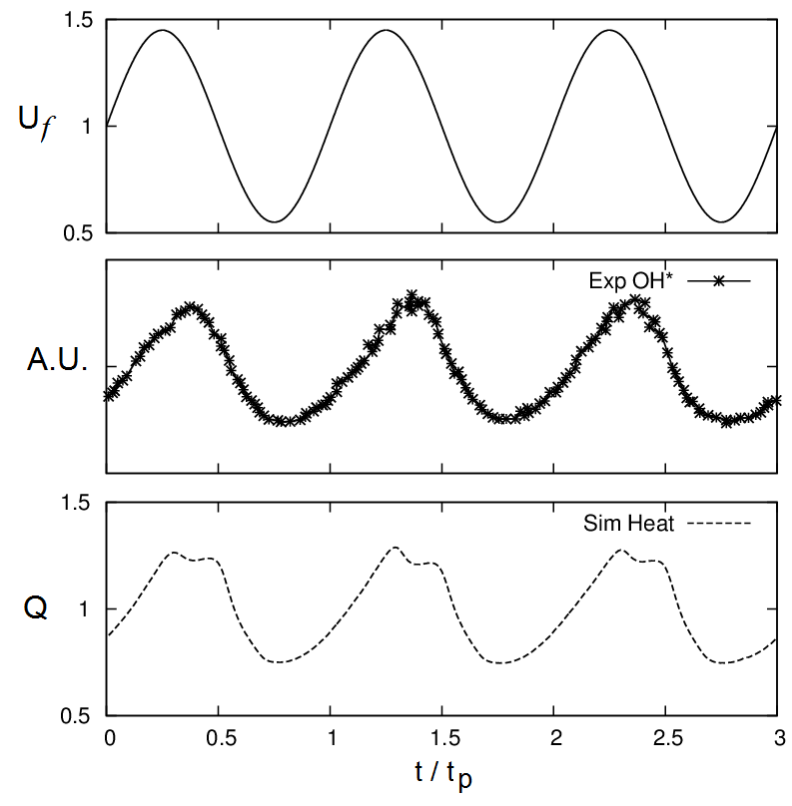

Fig. 7. Time series of signals with sinusoidal forcing at $\mathrm{f}=160 \mathrm{~Hz}$ and $\mathrm{A}=0.45$. Reference velocity normalised by the unforced bulk velocity (top), $\mathrm{OH}^{*}$ chemiluminescence signals in arbitrary unit (Balachandran et al. 2005) (middle) and heat release rate from simulations with USC mechanism normliased by its unforced value (bottom). 

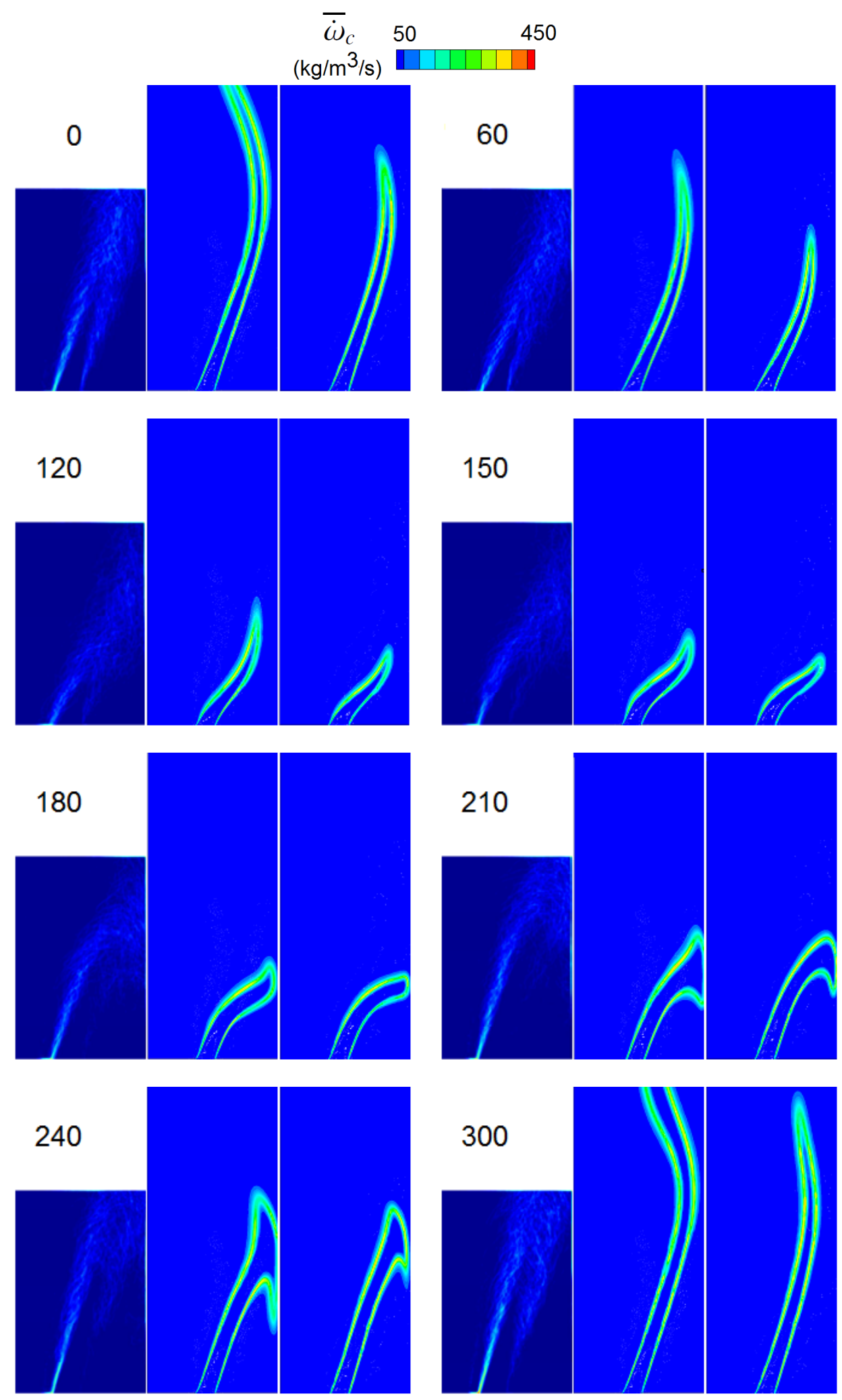

Fig. 8. Comparison of experimentally observed and computed flame shape at various phase angle for $f=40 \mathrm{~Hz}$ and $A=0.60$. The FSD for the experiment is shown (left), mean reaction rate is shown for the simulation using USC (middle) and UCSD (right) mechanisms. 

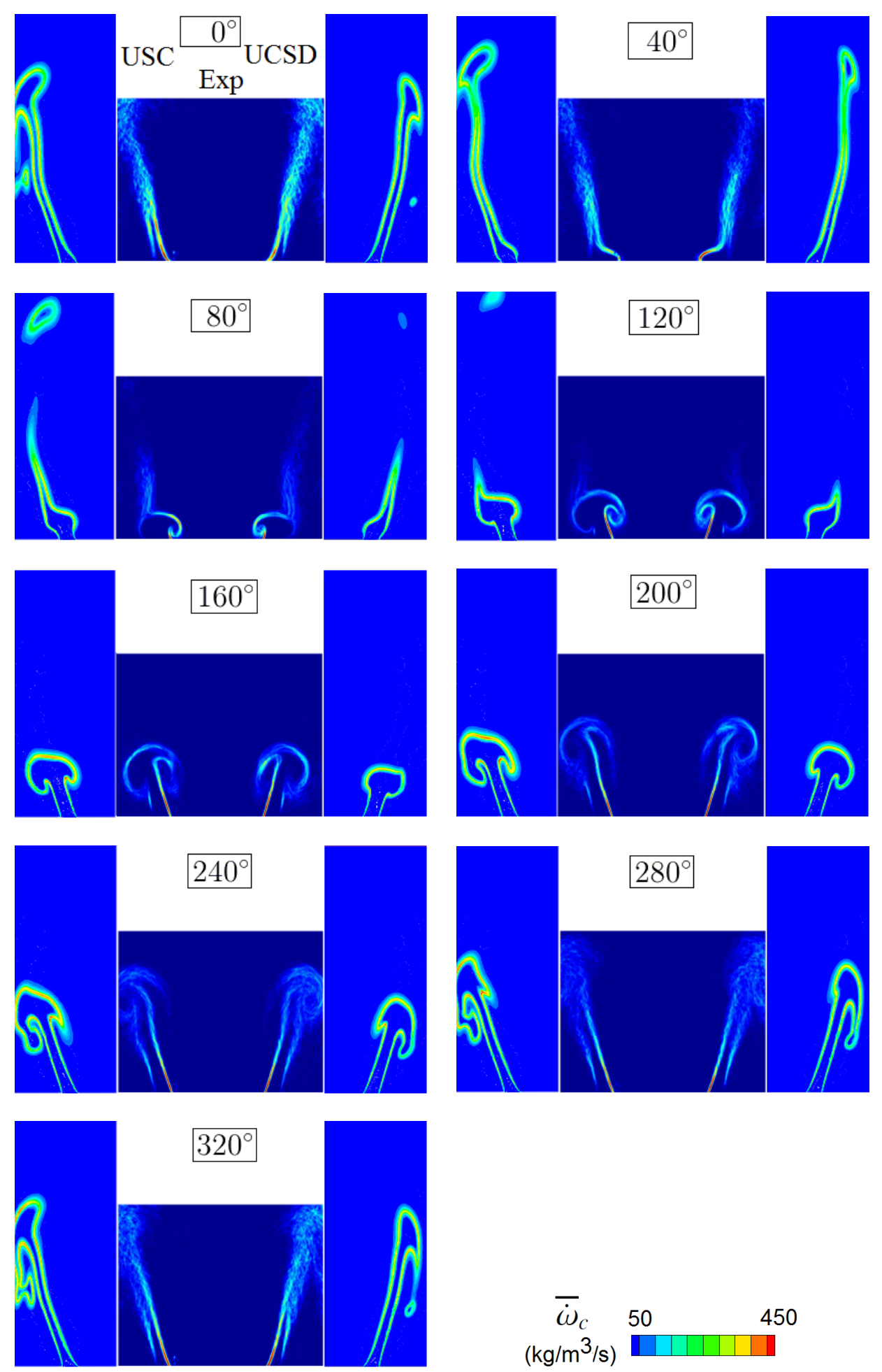

Fig. 9. Comparison of experimentally observed and computed flame shape at various phase angle for $f=160 \mathrm{~Hz}$ and $A=0.64$. The FSD for the experiment is shown (middle), mean reaction rate is shown for the simulation using USC (left) and UCSD (right) mechanisms. 

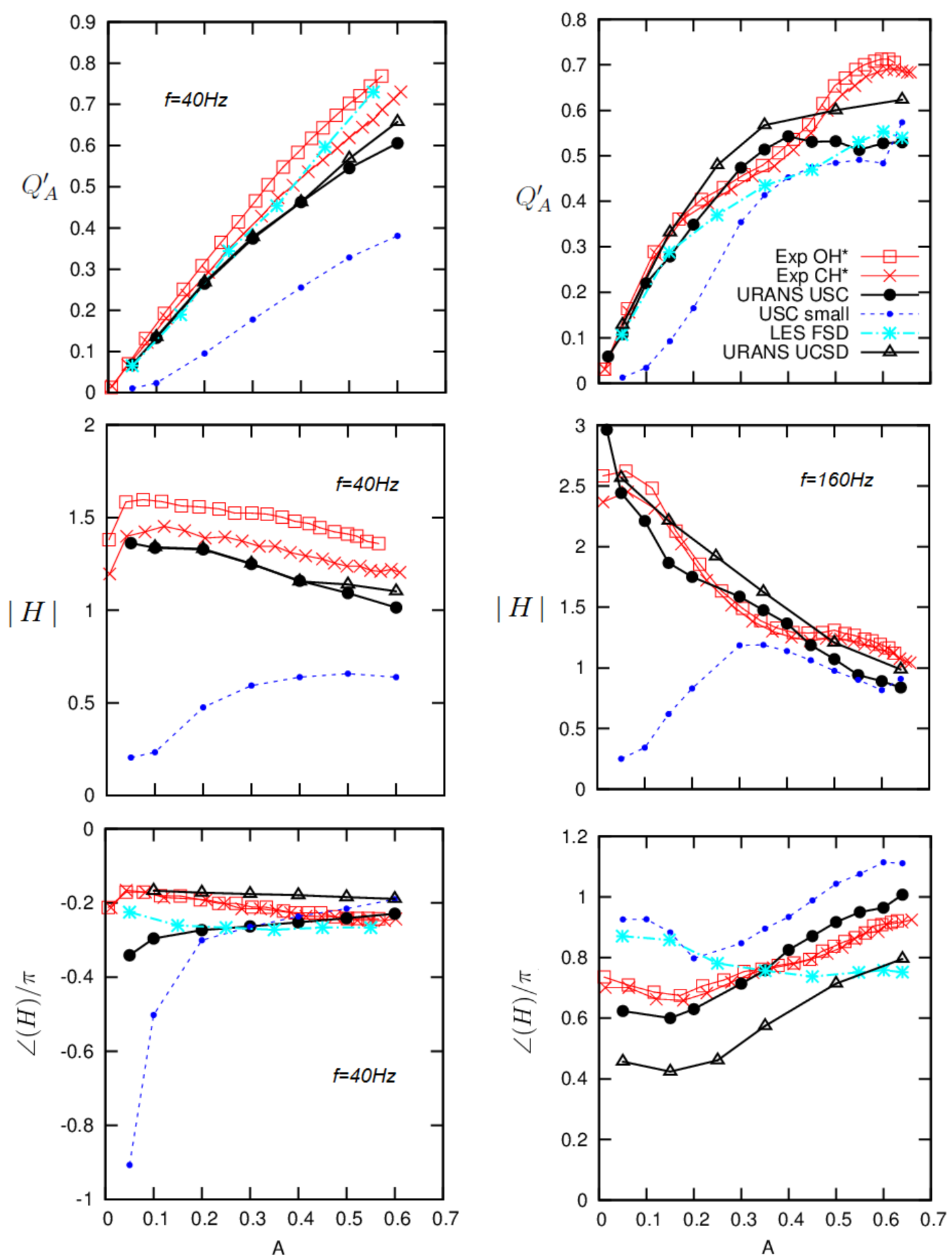

Fig. 10. Global Flame Describing Function against forcing amplitude $A$ : Flame response amplitude (top), Flame Describing Function magnitude (middle), Flame Describing Function phases (bottom) for $40 \mathrm{~Hz}$ (left) and $160 \mathrm{~Hz}$ (right) respectively."USC small” denotes results obtained from USC mechanism with a small domain corresponding to the PLIF window. "LES FSD" is the results of Han and Morgans (2015). 

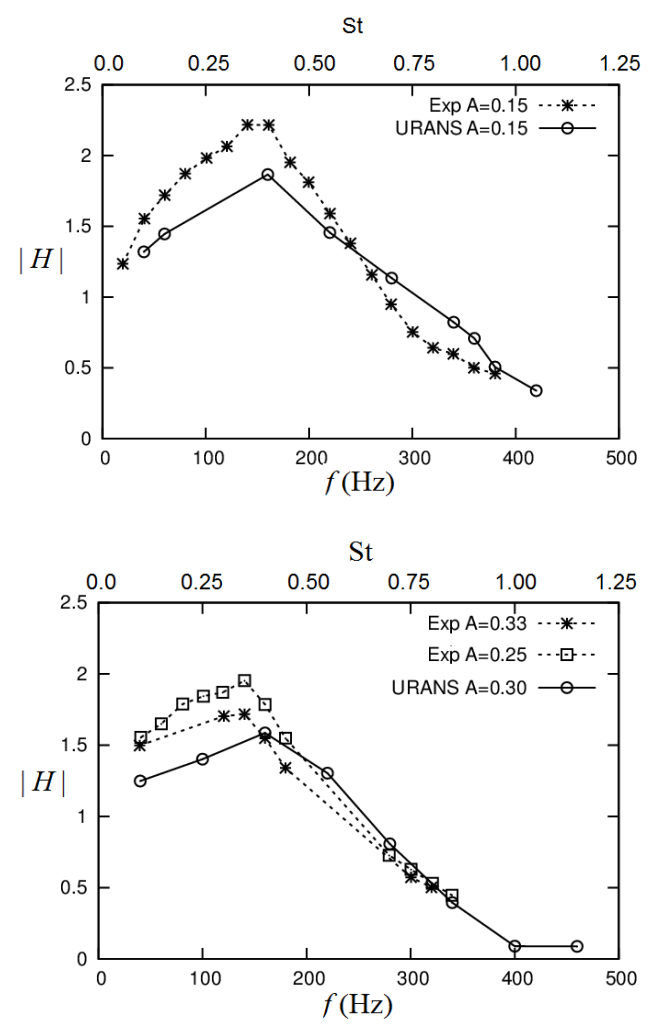

Fig. 11. Comparison of global FTF magnitude with forcing frequency $f$ and Strouhal number: (a) $A=0.15$, (b) $A=0.30$ between experiment and simulation result obtained from USC mechanism.

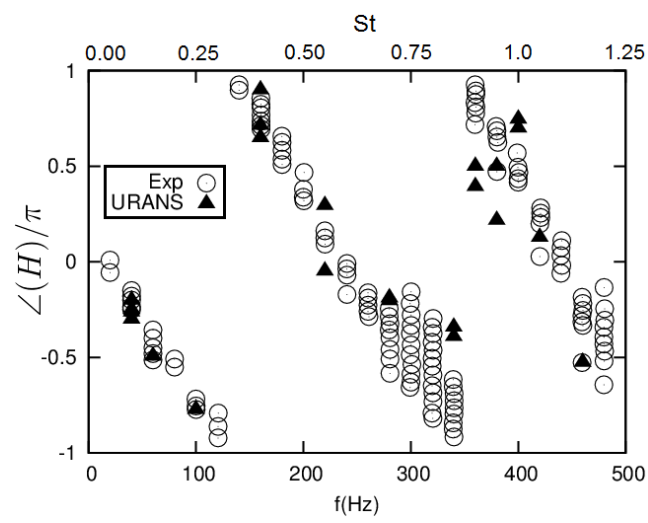

Fig. 12. Comparison of global FTF phase against forcing frequencies $f$ and Strouhal number between experiment and simulation results. 